\title{
New Worlds Observer Formation Control Design Based on the Dynamics of Relative Motion
}

\author{
Richard J. Luquette* \\ NASA Goddard Space Flight Center, Greenbelt, MD 20771
}

\begin{abstract}
The New Worlds Observer (NWO) mission is designed for the direct detection and characterization of extrasolar planets. The NWO mission concept employs a two spacecraft leader-follower formation on a trajectory around the Earth/Moon-Sun $L_{2}$ Libration Point. The leader spacecraft is baselined as a 4 meter optical telescope. The follower, Starshade spacecraft, is designed to suppress light from a central body star permitting direct detection of a surrounding exoplanetary system. The current design requires a nominal leader-follower separation range of 72 Megameters. NWO poses many challenges including formation control. NWO cycles between three principal control modes during the nominal mission timeline: science (fine pointing), realignment and transition. This paper examines formation control strategies in the context of dynamics of relative motion for two spacecraft operating in the vicinity of the Earth/Moon-Sun $L_{2}$ libration point. The paper presents an overview of the equations of relative motion followed by a discussion of each of the control modes. Discussion and analysis characterize control strategies for each of the mission control modes, including requirements, implementation challenges and project fuel budgets.
\end{abstract}

\section{Nomenclature}

$\begin{array}{ll}\boldsymbol{A}(t) & =\text { Dynamics matrix for linear form of equations of motion } \\ \boldsymbol{r}_{*} & =\text { Position vectors, subscripts depicted in figures } \\ \boldsymbol{e}_{\boldsymbol{E} L} & =\text { Unit vector from Leader Spacecraft to Earth/Moon barycenter } \\ \boldsymbol{e}_{S L} & =\text { Unit vector from Leader Spacecraft to Sun } \\ \boldsymbol{u}_{t h r u s t, L} & =\text { External control force applied to Leader spacecraft } \\ \boldsymbol{x} & =\text { Position of Follower referenced to Leader position } \\ { }^{I} v & =\text { Superscript designating inertial (I) frame } \\ \mu_{i} & =\text { Gravitational parameter for } i^{t h} \text { body of n-body system } \\ \|\boldsymbol{x}\| & =\text { The 2-norm of the vector } \boldsymbol{x}\end{array}$

\section{Introduction}

NASA's 2006 Strategic Plan outlines a Vision for Space Exploration. ${ }^{2}$ Among the varied challenges, the ten year plan incorporates the search for Earth-like planets around other stars. New Worlds Observer (NWO) is one of various mission concepts designed to discover and characterize Earth-like planets, and more generally extrasolar planets (exoplanets). ${ }^{1}$

The NWO mission concept includes two spacecraft, a Telescope and a Starshade. In this paper the term 'Telescope' refers to the complete Telescope spacecraft. The Telescope is designed to observe a planetary system about a star. The Starshade is equipped with a star shade designed to suppress light from the central star allowing observation of much dimmer orbiting planets. The formation flying aspect of NWO embodies several key technical challenges. Guidance, navigation and control systems must support maneuvering the Starshade into alignment with the Telescope/target star line-of-sight at the desired separation range. The formation holds alignment during the science observation. This process repeats for a planned sequence of

*Propulsion Branch, Code 597, Email: rich.luquette AT nasa.gov 


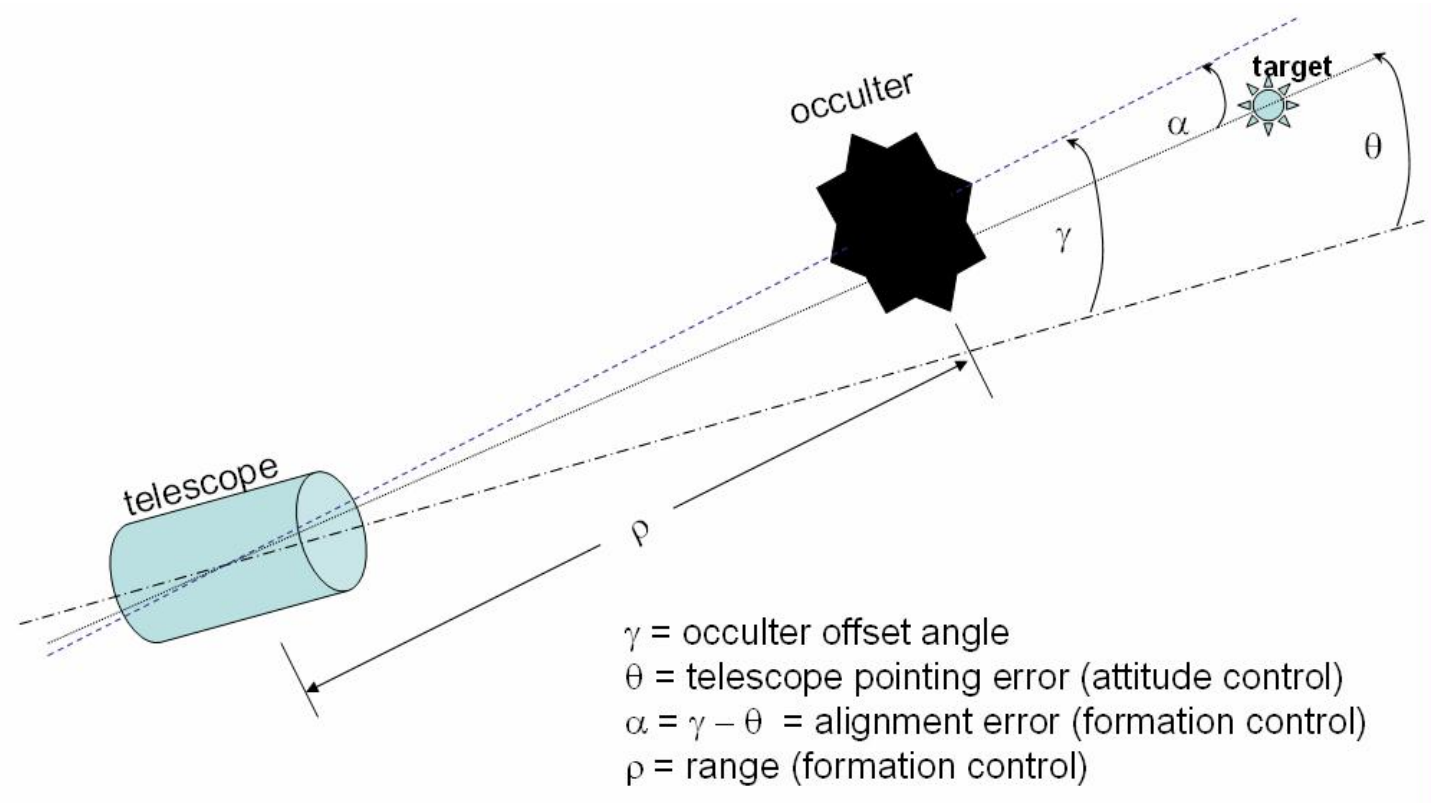

Figure 1. New Worlds Observer Formation Architecture: Two Spacecraft Orbiting the Earth/Moon Sun $L_{2}$ Point

target stars. Nominally, the formation sweeps an arc of 20 degrees during the transition between targets over a period of two weeks. Science observations average 1-2 days in duration per target.

Noecker ${ }^{4}$ and Leitner ${ }^{3}$ discuss various aspects of the navigation and control system for a Telescope/Starshade formation architecture. Their works explore technical requirements and solutions for accomplishing formation navigation and control. Additionally, the current author recently characterized the dynamics of relative motion of a formation under the influence of an n-body gravitational field. ${ }^{9}$

While the paper centers on the NWO mission, the methodology applies to analysis for any similar formation architecture.

\section{Mission Profile}

New Worlds Observer (NWO) employs a two spacecraft formation, depicted in Figure 1. The current design combines a 4 meter telescope with a 50 meter (diameter) starshade. The starshade incorporates a special petal pattern around its perimeter. The petals diffract light from the observation target onto the Telescope. The starshade suppresses light from the target star and passes light from the surrounding planetary system. The optical design allows direct imaging of exoplanetary systems. The mission is designed for imaging the habitable zone surrounding the star. The habitable zone is the region surrounding a star with conditions compatible for an Earth-like planet. Imaging of a target star will nominally last 1-2 days. Realignment of the formation for a new target will nominally require 2 weeks. The Telescope spacecraft is available for general astronomy during the realignment maneuver. The baseline mission design requires observation of 100 target stars over a five year period with the expectation of repeated visits to select targets. Additionally, the Telescope has a ten year design life, enabling for general astronomy for an additional five years after conclusion of the mission formation flying phase. 


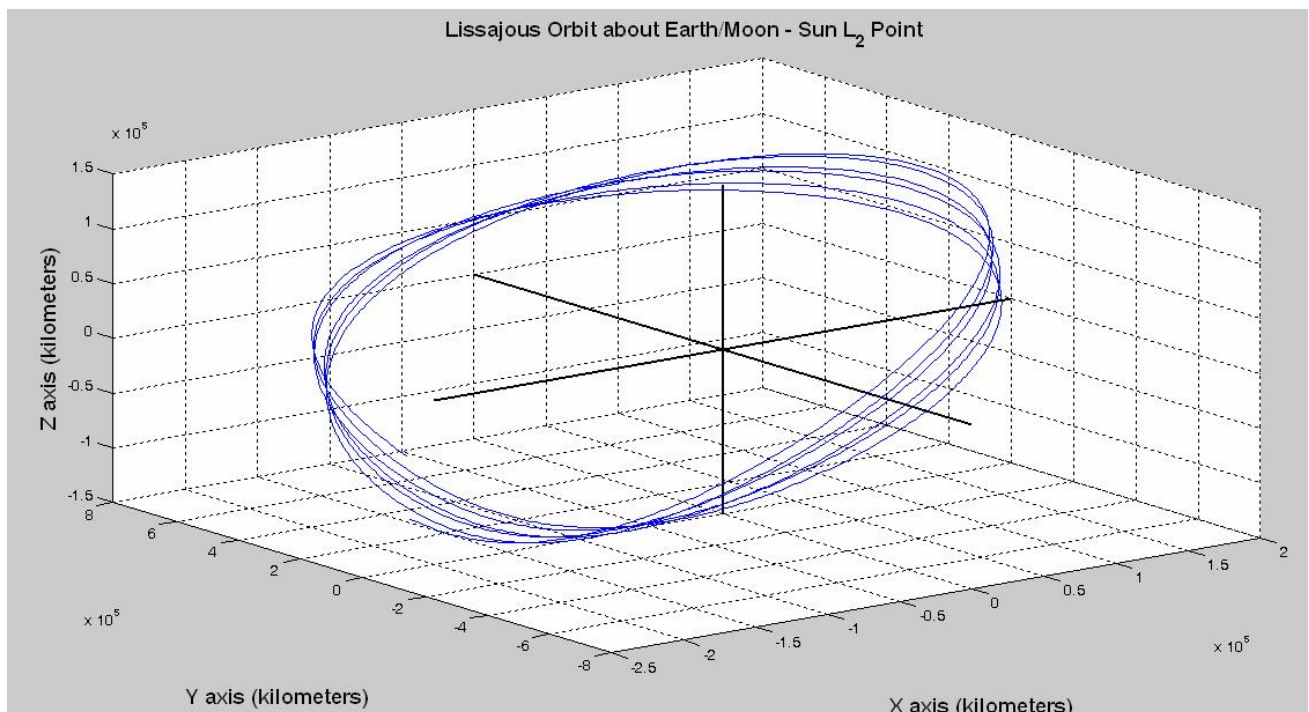

Figure 2. Leader (Telescope) Spacecraft Trajectory (typical) about Earth/Moon-Sun $L_{2}$ point

\section{A. Spacecraft Design}

The optical performance requirement of NWO drives an interdependency between the telescope aperture, the star shade diameter and the formation separation range. The current baseline design specifies a 4 meter telescope, a 50 meter diameter star shade, and a separation range of $72 \mathrm{Mm}$. The Telescope follows a nominal mission trajectory, typical of any single spacecraft mission operating near $L_{2}$. The Starshade is equipped to maneuver relative to the Telescope, managing the formation. Relative navigation instrumentation is distributed on both vehicles. In general key technical challenges are associated with the Telescope instrument, the star shade and formation flying. This paper concentrates on aspects of formation flying.

\section{B. Mission Trajectory}

The Telescope will be injected into a lissajous orbit about the Earth/Moon-Sun $L_{2}$ point, Figure 2. Excluding the period between launch and $L_{2}$ orbit injection, experience dictates the Telescope fuel requirement is approximately 1-4 meters/second/year for stabilizing the lissajous orbit. As the Telescope is designed for a 10 year mission, the total fuel requirement is not expected to exceed 40 meters/second. Trajectory maintenance maneuvers for the Telescope are infrequent, separated by intervals of six months or less. The Starshade matches Telescope trajectory maintenance maneuvers during the formation realignment mode, discussed later.

\section{Formation Configuration}

As the Telescope spacecraft travels along a stable manifold about the Earth/Moon-Sun $L_{2}$ point, the Starshade tracks the Telescope spacecraft holding a nominal offset of $72 \mathrm{Mm}$. During science observations the Starshade maintains a fixed inertial line-of-sight with respect to the Telescope spacecraft. For a nominal reconfiguration maneuver the Starshade slews the line-of-sight 20 degrees in two weeks, equivalent to approximately 1.4 degrees per day. With a stable trajectory about $L_{2}$ the Starshade would orbit the Telescope with a six month period, equal to 2 degrees per day. Reason concludes the Starshade trajectory does not generally align with a stable manifold about $L_{2}$. Formation flying analysis is best performed in a heliocentric inertial frame, based on the requirement to hold the formation inertially fixed during science. The Telescope follows a perturbed heliocentric orbit while the Starshade follows in formation. The restricted three-body rotating frame is useful for managing the Telescope trajectory, but less suitable for formation flying analysis since target star locations rotate in this frame. 


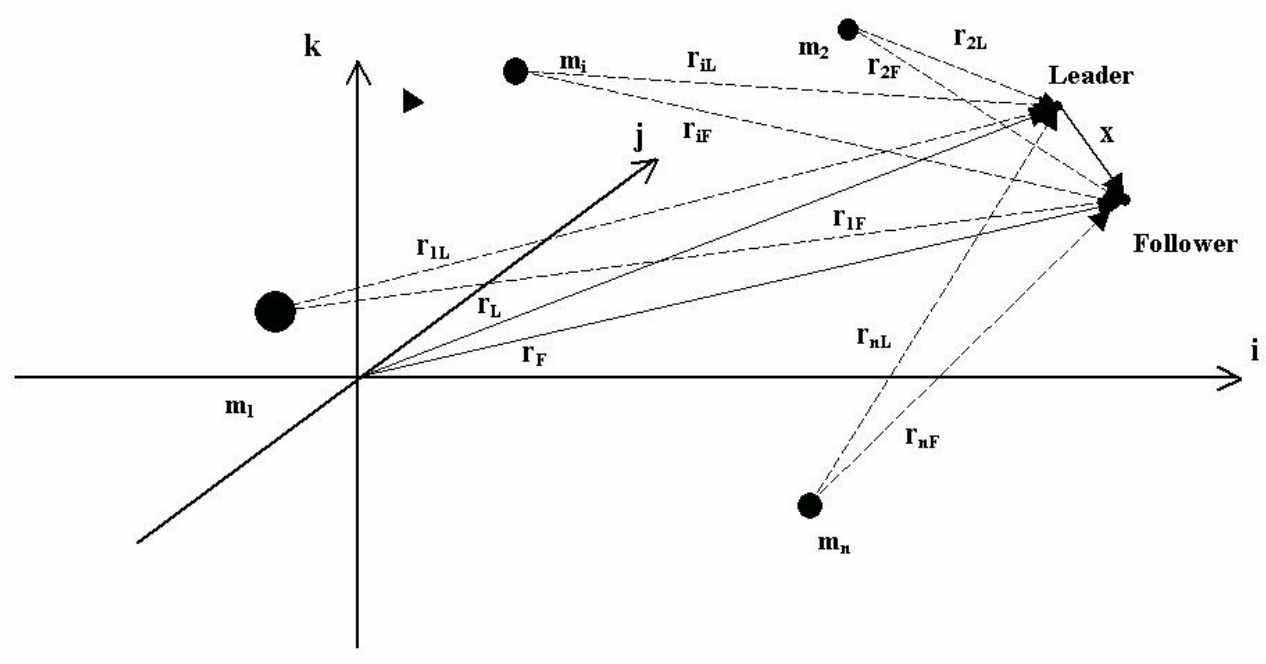

Figure 3. Two Spacecraft Under the Gravitational Influence of n-Bodies

\section{Relative Gravitational Dynamics}

Gravity and solar pressure are the principal external environmental forces acting on spacecraft operating near the Earth/Moon-Sun $L_{2}$ point. This paper limits analysis to the gravitational effects. Solar pressure, an important consideration, is difficult to quantify with limited maturity of spacecraft and mission design. Solar pressure effects are governed by spacecraft design and mission trajectories (orbit and attitude).

Relative gravitational dynamics are modeled in the context of a restricted n-body problem, Figure 3. 'Restricted' implies the spacecraft mass has negligible effect on the gravity field.

\section{A. Dynamics of Relative Motion for the n-Body Problem}

This section outlines formulation of the equations of relative motion based on a restricted n-body problem. Detailed development is presented in references $7-9$.

Expressed in inertial coordinates the equation for relative motion of a Follower spacecraft relative to a Leader in an n-body gravity field is:

$$
\begin{aligned}
\ddot{\boldsymbol{x}} & =\ddot{\boldsymbol{r}}_{\boldsymbol{F}}-\ddot{\boldsymbol{r}}_{\boldsymbol{L}} \\
& =-\sum_{i=1}^{n} \mu_{i} \frac{\boldsymbol{r}_{\boldsymbol{i F}}}{\left\|\boldsymbol{r}_{\boldsymbol{i} \boldsymbol{F}}\right\|^{3}}-\left(-\sum_{i=1}^{n} \mu_{i} \frac{\boldsymbol{r}_{i L}}{\left\|\boldsymbol{r}_{i L}\right\|^{3}}\right) \\
& =-\left\{\sum_{i=1}^{n} \frac{\mu_{i}}{\left\|\boldsymbol{r}_{\boldsymbol{i} \boldsymbol{F}}\right\|^{3}}\right\} \boldsymbol{x}-\sum_{i=1}^{n} \mu_{i}\left\{\frac{1}{\left\|\boldsymbol{r}_{\boldsymbol{i}}\right\|^{3}}-\frac{1}{\left\|\boldsymbol{r}_{i L}\right\|^{3}}\right\} \boldsymbol{r}_{i L}
\end{aligned}
$$

Note vectors $\boldsymbol{r}_{\boldsymbol{i L}}$ and $\boldsymbol{r}_{\boldsymbol{i} \boldsymbol{F}}$ as depicted in Figure 3 .

\section{B. Linear Parametric Form of Relative Dynamics}

The interspacecraft range is assumed small relative to ranges between the spacecraft and primary masses. Following manipulation, Equation 1 is expressed in linear parametric form: 


$$
\ddot{\boldsymbol{x}}={ }^{\boldsymbol{I}} \boldsymbol{\Xi}(t) \boldsymbol{x} ; \quad{ }^{\boldsymbol{I}} \boldsymbol{\Xi}(t)=-\sum_{i=1}^{n} \frac{\mu_{i}}{\left\|\boldsymbol{r}_{\boldsymbol{i L}}\right\|^{3}}\left[\mathbf{I}_{\mathbf{3}}-3\left(\boldsymbol{e}_{\boldsymbol{i L}} \boldsymbol{e}_{\boldsymbol{i L}}{ }^{\top}\right)\right]
$$

Note that $\boldsymbol{e}_{i L}$ denotes a unit vector along $\boldsymbol{r}_{i L}$.

Allowing applied thrust to the Follower spacecraft the dynamics in matrix form are

$$
\dot{\boldsymbol{\xi}}={ }^{\mathrm{I}} \mathbf{A}(t) \boldsymbol{\xi}+\boldsymbol{B} \boldsymbol{u}_{\text {thrust }, \boldsymbol{F}}
$$

Where:

$$
\boldsymbol{\xi}=\left[\begin{array}{l}
\boldsymbol{x} \\
\dot{\boldsymbol{x}}
\end{array}\right] ; \quad{ }^{\mathrm{I}} \mathbf{A}(t)=\left[\begin{array}{ll}
\mathbf{0} & \mathbf{I}_{\mathbf{3}} \\
{ }^{\mathrm{I}} \boldsymbol{\Xi}(t) & \mathbf{0}
\end{array}\right] ; \quad \boldsymbol{B}=\left[\begin{array}{l}
\mathbf{0} \\
\mathbf{I}_{\mathbf{3}}
\end{array}\right]
$$

The term ${ }^{I} \boldsymbol{\Xi}(t)$ is dependent on the Leader position in the n-body field and the mass properties of the n-bodies. The natural evolution of the Leader position and motion within the n-body field is predictable in time. Therefore, Equations 2 and 3 are equivalently considered a linear time-varying form. These equations provide utility in understanding the dynamics of relative motion and control system design.

\section{Relative Drift}

A detailed discussion of relative spacecraft dynamics based on Equation 1 is presented in [9]. In summary, ${ }^{I} \boldsymbol{\Xi}(t)$ serves as a tool for characterizing the instantaneous acceleration of a Follower spacecraft relative to a Leader. For the NWO design the Telescope is the Leader. The Starshade is the Follower. Equation 1 is evaluated with two primary bodies, the Sun and the Earth/Moon, expressed as:

$$
\begin{aligned}
& \ddot{\boldsymbol{x}} \quad={ }^{\boldsymbol{I}} \boldsymbol{\Xi}(t) \boldsymbol{x} \\
& { }^{\boldsymbol{I}} \boldsymbol{\Xi}(t)=-\left[\frac{\mu_{E}}{\left\|\boldsymbol{r}_{E L}\right\|^{3}}+\frac{\mu_{S}}{\left\|\boldsymbol{r}_{S L}\right\|^{3}}\right] \mathbf{I}_{\mathbf{3}}+\frac{3 \mu_{E}}{\left\|\boldsymbol{r}_{\boldsymbol{E L}}\right\|^{3}}\left(\boldsymbol{e}_{\boldsymbol{E} L} \boldsymbol{e}_{\boldsymbol{E} L}{ }^{\top}\right)+\frac{3 \mu_{S}}{\left\|\boldsymbol{r}_{S L}\right\|^{3}}\left(\boldsymbol{e}_{\boldsymbol{S L}} \boldsymbol{e}_{S L}{ }^{\top}\right)
\end{aligned}
$$

Where: $\boldsymbol{e}_{\boldsymbol{E} \boldsymbol{L}}$ is the unit vector from Leader Spacecraft to Earth/Moon barycenter $\boldsymbol{e}_{S L}$ is the unit vector from Leader Spacecraft to Sun

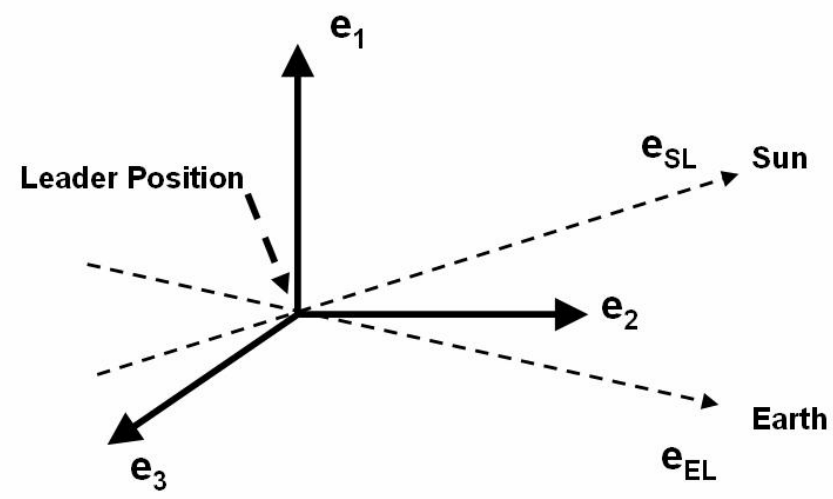

Figure 4. Orientation of Eigenvectors $\left(e_{1}, e_{2}, e_{3}\right)$ of ${ }^{I} \Xi\left(t_{0}\right)$ relative to $e_{S L}\left(t_{0}\right)$ and $e_{E L}\left(t_{0}\right)$ 


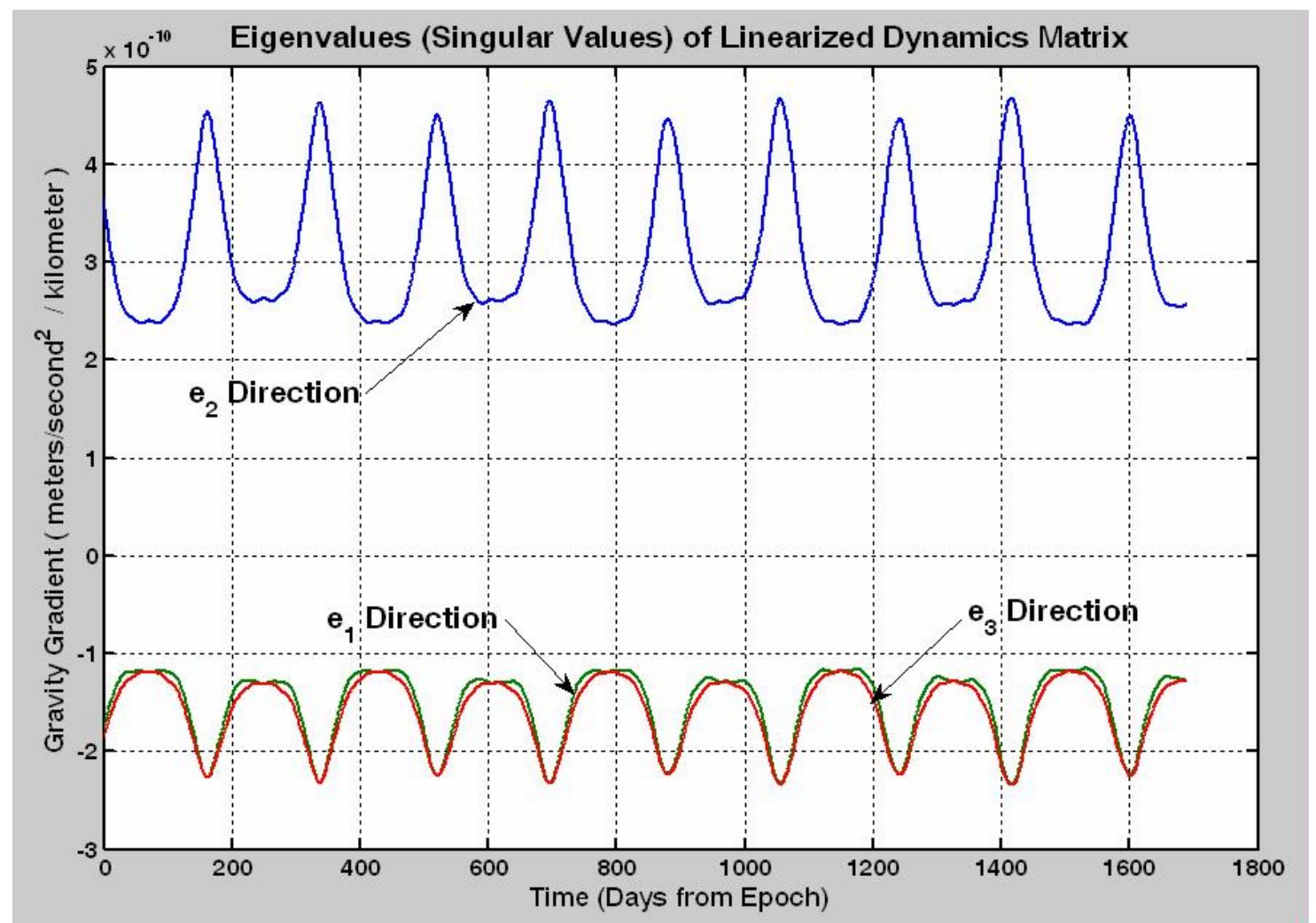

Figure 5. Magnitude of Gravity Gradient Along Eigenvectors, $e_{1}, e_{2}, e_{3}$

Based on Equation 4, the relative acceleration is conveniently resolved as components aligned with the instantaneous eigenvectors of ${ }^{I} \boldsymbol{\Xi}(t)$. The alignment of the eigenvectors is depicted in Figure 4. Since ${ }^{I} \boldsymbol{\Xi}(t)$ is symmetric, the eigenvectors are mutually orthogonal. $e_{2}$ and $e_{3}$ lie in the plane defined by the positions of the Sun, Earth/Moon barycenter and the Leader spacecraft. $e_{1}$ is normal to this plane. The associated eigenvalues define the relative acceleration per unit distance of separation, shown in Figure 5.

\section{Formation Control Strategies}

\section{A. Launch and Transit to $L_{2}$}

In brief, a single launch is envisioned to carry both NWO spacecraft into orbit. After launch the spacecraft will separate, allowing checkout during transit to the mission trajectory near Earth/Moon - Sun $L_{2}$. Maneuvers during the transit period will separate the spacecraft to the desired separation range, $72 \mathrm{Mm}$. Details of the mission profile from launch to injection around $L_{2}$ are under development. This stage is complete after both spacecraft complete checkout, and the Telescope is established on the mission trajectory about $L_{2}$. The Starshade follows on a controlled trajectory nominally aligned for the first science observation.

\section{B. Mission Stage}

The mission stage is baselined as a five year period of science observations. This stage is characterized by a cycle of three formation control modes: science, realignment and transition. Formation control modes are represented in Figure 6. A description of the control strategy for each mode follows. 


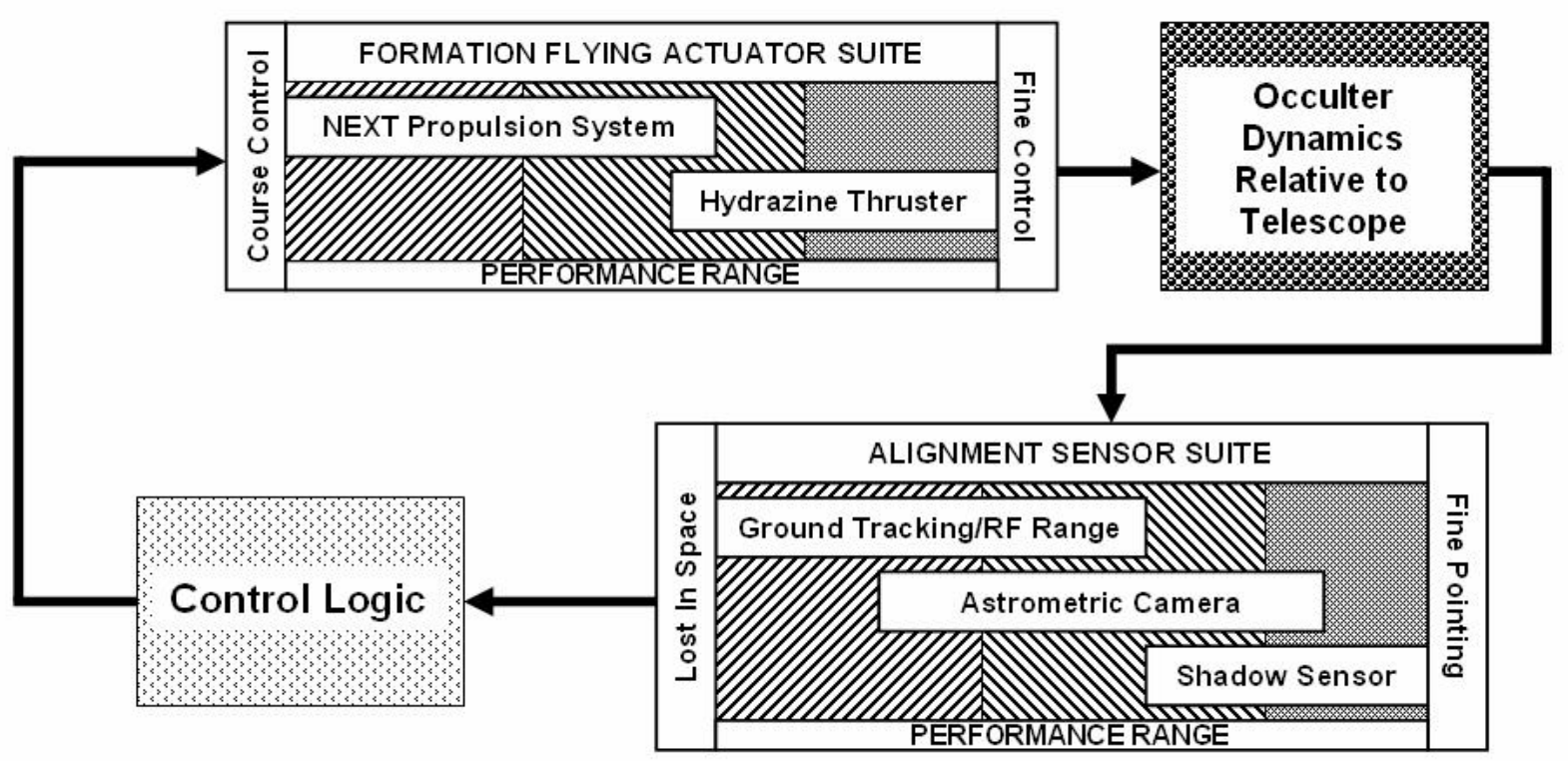

\section{Realignment Mode}

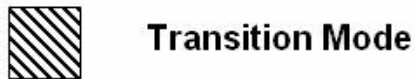

Science Mode

Figure 6. Formation Flying Alignment Control Architecture, $e_{1}, e_{2}, e_{3}$

\section{Science Mode}

The science mode controls formation alignment with a specific target star. The time required for collecting data on a given target varies in duration from hours to several days. Science observations serve to identify and characterize exoplanets. Science mode control is baselined to employ hydrazine thrusters to hold the Starshade within 1 meter of the Telescope/target star line-of-sight, equivalent to 3 milli-arcseconds (mas). The baseline design includes a formation sensor designed to detect the Starshade shadow at the Telescope. The formation sensor provides the misalignment error signal to implement autonomous formation control. Transition into the science mode requires detection of the Starshade shadow at the Telescope. Based on current design concepts, the transition requires an alignment error of less than 30 milli-arcseconds, or within 10 meters of the Telescope/target star line-of-sight at $72 \mathrm{Mm}$ range. The range control requirement is relaxed by comparison with an allowable variation of 100 kilometers.

\section{Realignment Mode}

After completing science data collection for a given target, the formation requires realignment to the next target. Realignment is accomplished by maneuvering the Starshade while the Telescope maintains a lissajous orbit about $L_{2}$. The nominal maneuver slews the formation line-of-sight through an angle of 20 degrees over a period of two weeks. During the slew relative navigation is supported by an astrometric camera affixed to the Starshade. The astrometric camera tracks the Telescope relative to the background stars. The Telescope will be equipped with beacons or utilize reflected sunlight for identification by the astrometric camera. Relative navigation is also supported by ground tracking and RF based interspacecraft ranging. The slew maneuver starts with a long duration thrust period to establish the desired relative velocity between the Starshade and the Telescope. The thrust period is followed by a coast period and culminates with a braking maneuver to slow the Starshade to a position required for the next target. The NEXT ${ }^{6}$ electric propulsion system is baselined for the slew maneuver. The realignment mode does not require fully autonomous control. 


\section{Transition Mode}

As the Starshade approaches the desired alignment with the next target the control system enters a transition mode. During this period of time the control system will employ electric propulsion and hydrazine to guide the Starshade to the entry criteria for the science mode. During the transition relative navigation is derived from astrometric camera measurements. Autonomous control is required during the transition period. Details of the control architecture including the transition from electric propulsion to hydrazine have yet to be developed.

\section{Control Design Analysis}

This section provides control system design analysis based on the relative dynamics presented in Section III. The Telescope follows the specific trajectory depicted in Figure 2. The Starshade/Telescope separation is maintained at $72 \mathrm{Mm}$.

Referring to Figure 5, the gravity gradient is nearly identical along $\boldsymbol{e}_{1}$ and $\boldsymbol{e}_{3}$, which for analysis are considered equal, represented as $\alpha(t)$. The gravity gradient along $\boldsymbol{e}_{2}$ is represented as $\gamma(t)$. Consider an arbitrary vector, $\boldsymbol{v}$, as the observation line-of-sight. $\boldsymbol{v}$ is expressed in terms of $\boldsymbol{e}_{1}, \boldsymbol{e}_{2}$ and $\boldsymbol{e}_{3}$, as:

$$
\boldsymbol{v}=v_{1} \boldsymbol{e}_{1}+v_{2} \boldsymbol{e}_{\mathbf{2}}+v_{3} \boldsymbol{e}_{\mathbf{3}}
$$

Based on Equation 4, the associated relative acceleration is:

$$
\begin{aligned}
{ }^{\boldsymbol{I}} \boldsymbol{\Xi}(t) \boldsymbol{v} & =\alpha(t)\left[v_{1} \boldsymbol{e}_{\mathbf{1}}+v_{3} \boldsymbol{e}_{\mathbf{3}}\right]+\gamma(t) v_{2} \boldsymbol{e}_{\mathbf{2}} \\
& =\alpha(t)\left[v_{1} \boldsymbol{e}_{\mathbf{1}}+v_{2} \boldsymbol{e}_{\mathbf{2}}+v_{3} \boldsymbol{e}_{\mathbf{3}}\right]+[\gamma(t)-\alpha(t)] v_{2} \boldsymbol{e}_{\mathbf{2}} \\
& =\alpha(t) \boldsymbol{v}+[\gamma(t)-\alpha(t)] v_{2} \boldsymbol{e}_{\mathbf{2}}
\end{aligned}
$$

Define $\boldsymbol{e}_{v \perp}$ and $\boldsymbol{e}_{\boldsymbol{v} \|}$ as the corresponding orthogonal and parallel components of $\boldsymbol{e}_{2}$ with respect to $\boldsymbol{v}$.

Then:

$$
\begin{aligned}
& e_{2} \quad=\left\{e_{2} \bullet e_{v \perp}\right\} e_{v \perp}+\left\{e_{2} \bullet e_{v \|}\right\} e_{v \|} \\
& { }^{I} \boldsymbol{\Xi}(t) \boldsymbol{v}=\alpha(t)\|\boldsymbol{v}\| \boldsymbol{e}_{\boldsymbol{v} \|}+[\gamma(t)-\alpha(t)] v_{2}\left[\left\{\boldsymbol{e}_{2} \bullet \boldsymbol{e}_{\boldsymbol{v} \perp}\right\} \boldsymbol{e}_{v \perp}+\left\{\boldsymbol{e}_{\mathbf{2}} \bullet \boldsymbol{e}_{\boldsymbol{v} \|}\right\} \boldsymbol{e}_{\boldsymbol{v} \|}\right] \\
& =\left\{\alpha(t)\|\boldsymbol{v}\|+[\gamma(t)-\alpha(t)] v_{2}\left\{\boldsymbol{e}_{2} \bullet \boldsymbol{e}_{\boldsymbol{v} \|}\right\}\right\} \boldsymbol{e}_{\boldsymbol{v} \|}+[\gamma(t)-\alpha(t)] v_{2}\left\{\boldsymbol{e}_{\mathbf{2}} \bullet \boldsymbol{e}_{\boldsymbol{v} \perp}\right\} \boldsymbol{e}_{\boldsymbol{v} \perp}
\end{aligned}
$$

Equation 7 provides useful insight into the drift behavior of the Follower (Starshade) with respect to the Leader (Telescope). First, the relative acceleration is constrained to the plane defined by $\boldsymbol{v}$ and $\boldsymbol{e}_{\mathbf{2}}$. Second, the crosstrack drift term is zero when the observation line-of-sight is orthogonal to $\boldsymbol{e}_{2}$. The latter point is significant for the NWO mission. Sun constraints for NWO formation alignment nominally require orthogonality between the observation line-of-sight and the Telescope/Sun line. Therefore, the observation line-of-sight will tend toward orthogonality with $\boldsymbol{e}_{2}$, and minimized crosstrack drift.

As validation, the crosstrack drift was computed for the ephemeris depicted in Figure 2 with an inertially fixed observation vector. The observation vector was initialized to align with the Telescope/Sun line at the simulation start time. The separation range between spacecraft was $72 \mathrm{Mm}$. The magnitude of the crosstrack drift is shown in Figure 7. The angle between the crosstrack drift and the normal to the $\boldsymbol{v}, \boldsymbol{e}_{2}$ plane is shown in Figure 8. The results clearly show the crosstrack drift lies in (or near) the $\boldsymbol{v}, \boldsymbol{e}_{2}$ plane. Spikes in Figure 8 are attributed to computation noise associated with zero (or minimal) crosstrack drift. Note correlation with results in Figure 7 . The simulation employed a full planetary gravity model to compute relative acceleration (truth model). 


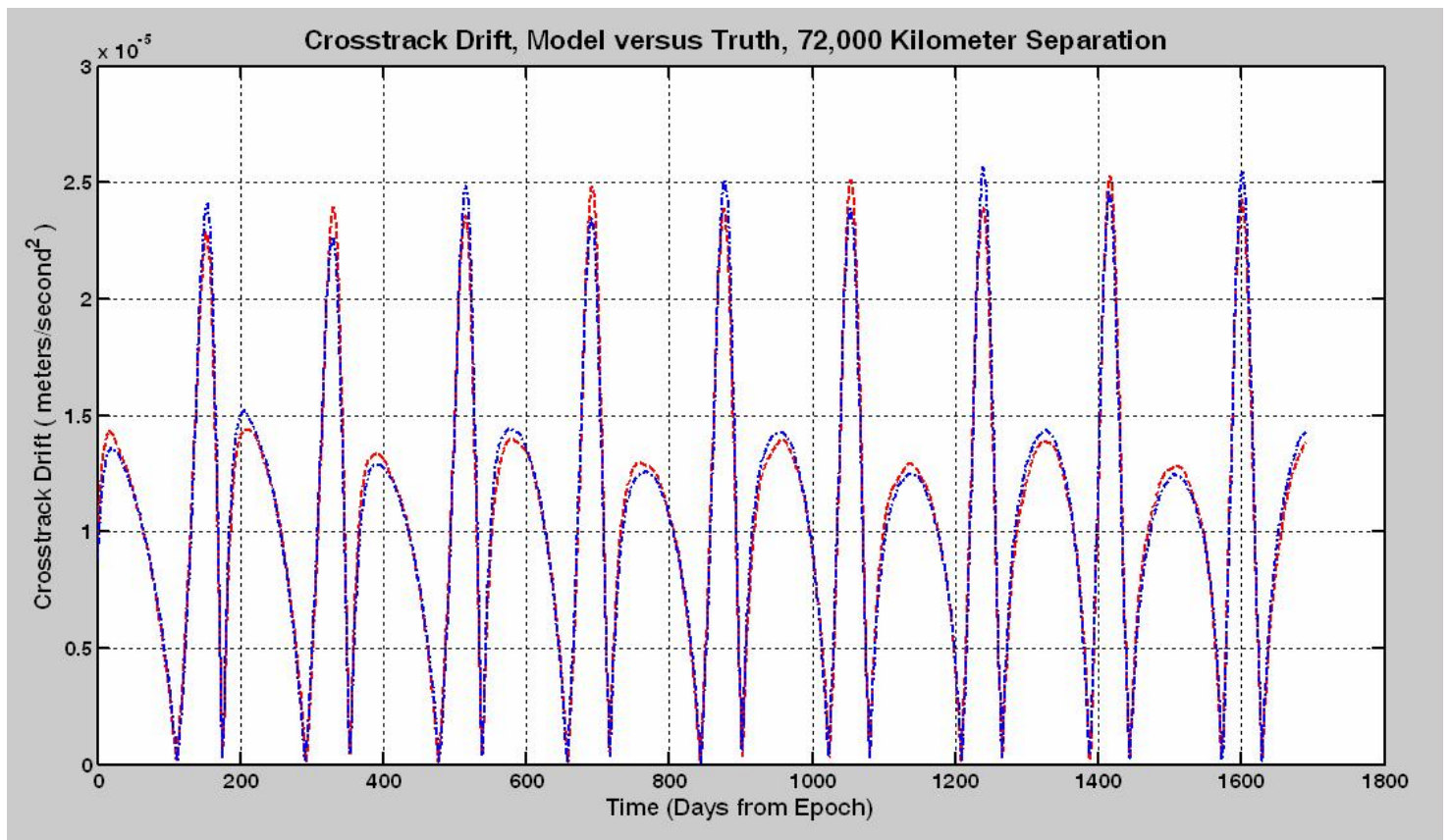

Figure 7. Crosstrack Drift Magnitude Variation Along Trajectory Timeline

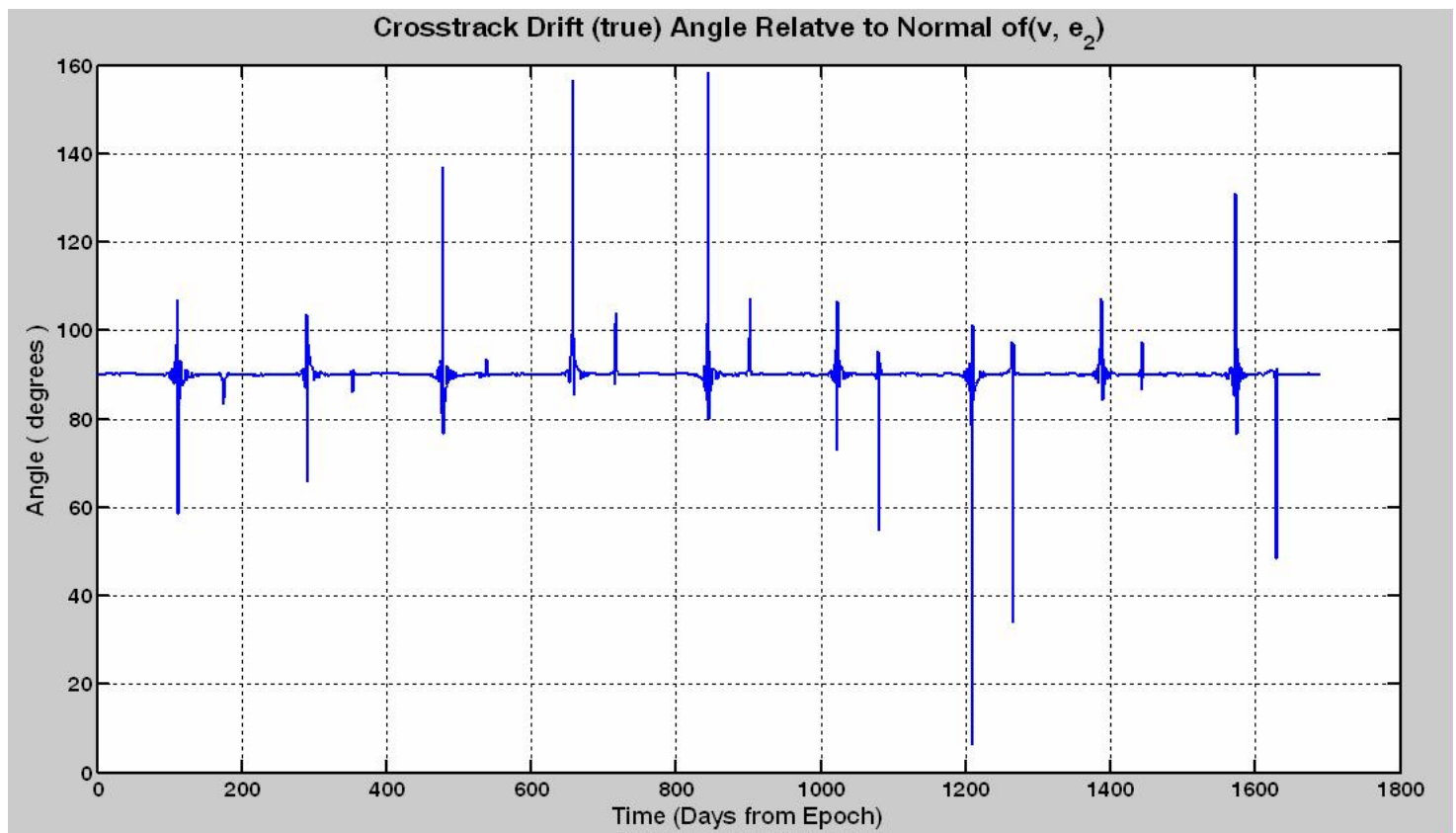

Figure 8. Angle Between Crossdrift Vector and Normal to $e_{2}, v$ Plane

In the case where $\boldsymbol{v}$ and $\boldsymbol{e}_{\mathbf{2}}$ are orthogonal, the crosstrack drift is zero and the radial drift is $\alpha(t)\|\boldsymbol{v}\|$. Based on a $72 \mathrm{Mm}$ separation and data from Figure 5, the maximum differential radial acceleration is approximately 0.00002 meters $/ \mathrm{sec}^{2}$. NWO requirements specify a control box of $+/-4,000$ kilometers along the radial direction. Assuming the Starshade is at rest at the most distant edge of the control box, the 
transit time to the opposite control box edge is approximately 7.3 days with a Starshade relative velocity of 12.6 meters $/$ sec. Therefore, maneuvers are not required to maintain the desired radial separation during science observations.

Crosstrack corrections are expected to be more frequent due to the tight pointing requirement. The allowable Starshade position offset from the Telescope/target line-of-sight is approximately 1 meter. From Equation 7 and analysis presented in Figure 7, the magnitude of the crosstrack drift is dependent on the Telescope position (defines orientation of $\boldsymbol{e}_{\mathbf{2}}$ ) and the orientation of the observation vector. Therefore, the control system requirement must be evaluated against a defined mission trajectory and correlated observation schedule.

\section{Summary/Conclusions}

This paper summarizes prior work on the development of a linear parameterized form of relative dynamics in the context of a restricted n-body problem. Relative dynamics are expressed in inertial coordinates. The utility of the analytic model is demonstrated through analysis of the baseline design for the proposed New Worlds Observer. The analysis is intended to provide the design engineer with a feel for control issues associated with managing formation maintenance. Detailed analysis is required for complete control system design. The reader is reminded that solar pressure effects, not addressed, must be evaluated for any formation design.

\section{References}

\footnotetext{
1 "The New Worlds Observer (NWO) Homepage," http://newworlds.colorado.edu/

2 "2006 NASA Strategic Plan,", National Aeronautics and Space Administration, 2006

${ }^{3}$ Leitner, J., "Formation Flying System Design for a Planet-Finding Telescope-Occulter System," UV/Optical/IR Space Telescope Innovative and Concepts III, Proc.of SPIE, Vol. 6687 (2007)

${ }^{4}$ Noecker, M. C., "Formation Flying System Design for a Planet-Finding Telescope-Occulter System," Techniques and Instrumentation for Detection of Exoplanets III, Proc.of SPIE, Vol. 6693 (2007)

${ }^{5}$ Luquette, R. J. and Sanner, R. M., "Linear State-Space Representation of the Dynamics of Relative Motion, Based on Restricted Three Body Dynamics," AIAA Guidance and Control Conference, August 2004, Paper No. AIAA-2004-4783.

${ }^{6}$ Pinero, L. R. and Sovey, J. S., "NEXT Thruster Component Verification Testing," NASA/TM2007-215036, December

${ }^{7}$ Luquette, R. J. and Leitner, J. and Gendreau, K. C. and Sanner, R. M., "Formation Control for the MAXIM Mission," 2nd International Symposium on Formation Flying Mission \& Technologies, September 2004.

${ }^{8}$ Luquette, R. J., "Nonlinear Control Design Techniques for Precision Formation Flying at Lagrange Points," Ph.D. Dissertation, Department of Aerospace Engineering, University of Maryland, College Park, MD, December 2006.

${ }^{9}$ Luquette, R. J. and Sanner, R. M., "Spacecraft Formation Contrl: Managing Line-of-Sight Drift Based on the Dynamics of Relative Motion, Based on Restricted Three Body Dynamics," 3rd International Symposium on Formation Flying Mission Es Technologies, April 2008.
} 2007. 